\title{
Incidence of fecal Enterobacteriaceae producing broad-spectrum beta-lactamases in patients with hematological malignancies
}

\author{
Milan Kolara, Miroslava Htoutou Sedlakovaa, Vendula Pudovaa, Magdalena Roderova ${ }^{b}$, \\ Jiri Novosada , Marketa Senkyrikova a, Romana Szotkowskac, Karel Indrak ${ }^{c}$
}

\begin{abstract}
Aim. Given the steadily increasing numbers of resistant bacteria, the frequency and severity of infections are on the rise. In patients with hematological malignancies, the treatment itself increases the risk of complicating bacterial infections. One important mechanisms of resistance is production of broad-spectrum beta-lactamases, increasingly detected not only in bacterial pathogens but also in bacteria contained in the normal microflora of the human body. The objectives of this study were determination and analysis of the prevalence of multiresistant ESBL-and AmpC-positive Enterobacteriaceae in the gastrointestinal tract (GIT) of patients with hematological malignancies.

Methods. For 3 months, rectal swabs were taken from patients with hematological malignancies and analyzed using chromogenic screening plates to isolate ESBL- and AmpC-producing Enterobacteriaceae. Beta-lactamase production was determined by phenotype tests and confirmed by detecting genes encoding ESBL and AmpC types. At the same time, ESBL- and AmpC-positive Enterobacteriaceae were isolated from clinical samples collected from patients with bacterial infection.

Results. Over the study period, fifteen patients (21\%) of all patients treated at the Department of Hemato-Oncology were shown to have ESBL- or AmpC-positive Enterobacteriaceae in their GIT. Most frequently identified were ESBLpositive strains of Klebsiella pneumoniae and AmpC-positive strains of Citrobacter freundii. The ESBL enzymes were mainly of the CTX-M type. Isolates producing AmpC were found to contain genes for enzymes mainly from the CIT and DHA groups.

Conclusion. The study identified patients diagnosed with urinary tract and bloodstream infections caused by ESBLpositive strain of Klebsiella pneumoniae and AmpC-positive strain of Enterobacter cloacae contained in the GIT microflora.
\end{abstract}

Key words: Enterobacteriaceae, ESBL, AmpC, fecal carriage

Received: March 10, 2014; Accepted: July 9, 2014; Available online: August 18, 2014

http://dx.doi.org/10.5507/bp.2014.042

${ }^{a}$ Department of Microbiology, Faculty of Medicine and Dentistry, Palacky University Olomouc, Czech Republic ' Institute of Molecular and Translational Medicine, Faculty of Medicine and Dentistry, Palacky University Olomouc 'Department of Hemato-Oncology, University Hospital Olomouc

Corresponding author: Milan Kolar, e-mail:milan.kolar@fnol.cz

\section{INTRODUCTION}

The immune system of patients with hematological malignancies is seriously weakened due to the myelosuppressive and immunosuppressive effects of cytostatic agents. As a result, their prognosis is negatively affected by potential bacterial infections that have been - and are very likely to continue to be - of major importance. One of the main reasons is the fact that a large proportion of these infections are of endogenous origin, causative agent being a part of the human microflora. Another factor, is the changing distribution of individual bacterial species. Until recently, Gram-positive bacteria were most prevalent. Today, Gram-negative bacteria are becoming increasingly serious, in particular Enterobacteriaceae and strains of Pseudomonas aeruginosa $a^{1-3}$.

Serious bacterial pathogens seen in patients with hematological malignancies include Enterobacteriaceae. In their study of patients with hematological malignancies, Cattaneo et al. reported strains of Escherichia coli to be the most frequent isolates (almost 25\%) in bacteremia ${ }^{4}$.
A multicenter study of bacteremia in patients with hematological malignancies treated in Czech and Slovak centers demonstrated that Enterobacteriaceae accounted for more than $50 \%$ of all isolates ${ }^{5}$. At the present time, the clinical significance of Enterobacteriaceae is significantly intensified by their increasing resistance to antimicrobial agents. In the case of beta-lactam antibiotics, the most important mechanism of resistance is production of ESBL and AmpC types of broad-spectrum beta-lactamases ${ }^{6,7}$.

Bacterial complications in patients with hematological malignancies pose a serious threat, particularly in the case of neutropenia. Effective initial antibiotic therapy for febrile neutropenia decreases mortality to $2-10 \%\left(\mathrm{ref}^{8}{ }^{8}\right)$. At present, however, there is a significantly increasing risk of mortality rising due to growing bacterial resistance and the associated failure of antibiotic therapy. For instance, patients with hematological malignancies have $25 \%$ higher mortality due to bacteremia caused by ESBL-positive Enterobacteriaceae and 69\% higher death rates in the case of positive carbapenemase production ${ }^{9,10}$.

It should be stressed that as an effect of the hospital 
environment, the bacterial microflora of patients with hematological malignancies is changed from natural primary to secondary, with a higher proportion of multiresistant bacteria posing a risk because of a role they may play in the etiology of complicating bacterial infections. Arnan et al. documented that $29 \%$ of neutropenic patients carried ESBL-positive Escherichia coli strains, compared with only $14 \%$ on admission ${ }^{11}$.

The aims of this study were determining the prevalence of carrier multiresistant ESBL- and AmpC-positive Enterobacteriaceae in patients with hematological malignancies, their molecular genetic analysis and assessment of their clinical significance. The study was the first of its kind in the Czech Republic.

\section{MATERIAL AND METHODS}

Over 3 months (1 November 2012 - 31 January 2013), rectal swabs were taken from all patients treated at the Department of Hemato-Oncology, University Hospital Olomouc, Czech Republic. From the samples, Enterobacteriaceae were isolated using chromogenic screening plates for the detection of ESBL- and AmpCproducing strains (Brilliance ${ }^{\mathrm{TM}}$ ESBL Agar, Oxoid). At the same time (plus an additional month), clinical samples (blood, urine, other body fluids and exudates, lower airway samples and intravascular catheters) collected from patients with febrile neutropenia were investigated for ESBL- and AmpC-positive Enterobacteriaceae causing complicating bacterial infections.

Each isolate was identified by standard microbiological techniques with the Phoenix ${ }^{\mathrm{TM}}$ automated system (BD Diagnostics) and MALDI Biotyper system (Bruker Daltonics) (ref. $\left.{ }^{12}\right)$. The strains were selected in such a manner that from each patient, a single strain of each species isolated as the first one was included.

Phenotypic detection of ESBL and AmpC beta-lactamases was carried out using a modified double-disk synergy test and a modified AmpC test ${ }^{13}$. In all positive isolates, polymerase chain reaction (PCR) detected the presence of genes encoding relevant betalactamases $^{14-18}$.

All isolates were analyzed by comparing wholegenome DNA with pulsed-field gel electrophoresis (PFGE) $\left(\right.$ ref. $\left.^{19}\right)$.

\section{RESULTS}

Over the study period, a total of 71 patients were enrolled. The most frequent diagnoses were acute myeloid leukemia, acute lymphoblastic leukemia, chronic lymphocytic leukemia, chronic myelomonocytic leukemia, multiple myeloma, Hodgkin lymphoma, diffuse large B-cell lymphoma and other non-Hodgkin lymphoma. There were 43 males and 28 females with ages ranging from 21 to 76 years. The mean age was 54 years; the median age was
58 years. Over the study period, febrile neutropenia was diagnosed in 46 patients $(64.8 \%)$.

A total of 15 patients $(21.1 \%)$ were demonstrated to have Enterobacteriaceae producing broad-spectrum betalactamases in their gastrointestinal tract (GIT). ESBLand AmpC-positive Enterobacteriaceae were found in 8 (11.3\%) and 7 (9.8\%) patients, respectively.

Of the 15 patients carrying ESBL- and AmpC-positive Enterobacteriaceae in their GIT, nine (60.0\%) had febrile neutropenia. In the group of the 56 patients (no ESBLand AmpC-positive strains detected in the GIT), febrile neutropenia was noted in $66.1 \%$ of subjects. Thus, carriage of Enterobacteriaceae producing broad-spectrum beta-lactamases in the GIT was not shown to be a risk factor for the development of febrile neutropenia.

Among the 15 patients with the GIT colonized by producers of broad-spectrum beta-lactamases, only one was hospitalized for the first time. The mean length of hospital stay was 25 days (range, 3-70 days). Thirteen patients $(86.7 \%)$ had been hospitalized at the Department of Hemato-Oncology in the preceding six months (previous hospital stays ranging from 3 to 58 days; a mean of 21 days).

The 8 cases of ESBL-positive Enterobacteriaceae comprised strains of Klebsiella pneumoniae (75.0\%), Escherichia coli (12.5\%) and Enterobacter aerogenes (12.5\%). The AmpC-positive bacteria were Citrobacter freundii (42.8\%), Enterobacter cloacae (28.6\%), Escherichia coli $(14.3 \%)$ and Klebsiella pneumoniae (14.3\%) strains.

PCR revealed the presence of a gene for beta-lactamase from the CTX-M-1-like group in 7 ESBL-positive isolates $(87.5 \%)$, the $b l a_{\mathrm{SHV}}$ gene was detected in 6 cases $(75.0 \%)$ and the $b l a_{\mathrm{TEM}}$ gene was noted in all ESBLpositive isolates. Isolates producing $\mathrm{AmpC}$ were found to contain genes for enzymes from the CIT, DHA and EBC groups. The species composition of ESBL- and AmpC-positive Enterobacteriaceae and genes encoding

Table 1. ESBL-positive Enterobacteriaceae isolated from GIT of 71 patients and detected genes for beta-lactamases.

\begin{tabular}{lcl}
\hline Species & $\begin{array}{c}\text { No. of } \\
\text { isolates }\end{array}$ & Beta-lactamase type \\
\hline Enterobacter aerogenes & 1 & CTX-M-1-like, TEM \\
Escherichia coli & 1 & CTX-M-1-like, TEM \\
Klebsiella pneumoniae & 1 & SHV, TEM \\
Klebsiella pneumoniae & 5 & CTX-M-1-like, \\
& & SHV, TEM \\
\hline
\end{tabular}

Table 2. AmpC-positive Enterobacteriaceae isolated from GIT of 71 patients and detected genes for beta-lactamases.

\begin{tabular}{lcl}
\hline Species & $\begin{array}{c}\text { No. of } \\
\text { isolates }\end{array}$ & Beta-lactamase type \\
\hline Citrobacter freundii & 2 & CIT \\
Citrobacter freundii & 1 & CIT, DHA \\
Enterobacter cloacae & 1 & EBC \\
Enterobacter cloacae & 1 & EBC, DHA \\
Escherichia coli & 1 & CIT \\
Klebsiella pneumoniae & 1 & DHA \\
\hline
\end{tabular}




\section{PFGE}

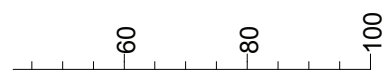

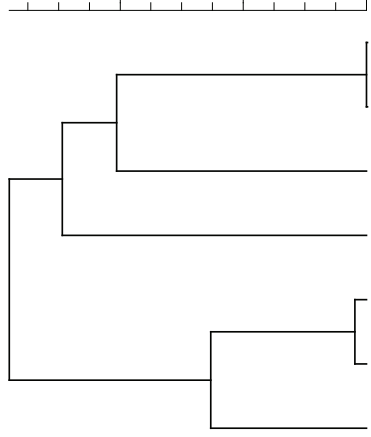

856

1342

20641

20344

20125

20446 765

horizontal axis - similarity of bacterial isolates (\%) vertical axis - isolate number, patient initials

Fig. 1. Dendrogram of Klebsiella pneumoniae isolates.

beta-lactamases detected in individual isolates are shown in Tables 1 and 2.

Comparison of whole-genome DNA with PFGE in Klebsiella pneumoniae revealed only one identical pair of isolates from two different patients and one pair of isolates with very similar restriction profiles, very likely corresponding to a single genetic change. This result is clearly shown as a dendrogram in Fig. 1. The similarity of the other isolates within individual species, in accordance with defined parameters - Dice (Tol 1.0\%-1.0\%) - was lower than $60 \%$ (dendrograms not shown).

Over the study period, two patients with a confirmed presence of ESBL- or AmpC-positive Enterobacteriaceae in the GIT were diagnosed with bacterial infections, one patient with urinary tract infection and the other with bloodstream infection. The etiological agents were ESBLpositive Klebsiella pneumoniae isolated from urine and AmpC-positive Enterobacter cloacae from blood. In both pathogenic strains, a restriction profile identical to that in GIT isolates was found.

\section{DISCUSSION}

Comprehensive treatment of patients with hematological malignancies increases the risk of complicating bacterial infections. Given the characteristics of these patients, antibiotic therapy must be initiated when the first signs of bacterial infection appear. There is no time to wait for the results of microbiological tests, including identification of the bacterial agent and determination of its susceptibility to antimicrobials.

Important pathogenic bacteria found in patients with hematological malignancies are Enterobacteriaceae producing both ESBL and AmpC broad-spectrum betalactamases. These multiresistant bacteria were found in the GIT microflora in patients with hematological malignancies ${ }^{15,20}$. Liss et al. documented that $17.5 \%$ of patients with hematological and oncological malignancies were colonized with ESBL-positive Enterobacteriaceae ${ }^{21}$.
This study demonstrated a $21 \%$ prevalence of ESBLand AmpC-positive Enterobacteriaceae carriage in the GIT of patients with hematological malignancies; at the same time, it confirmed the effect of previous hospital stays on positive carriage of these bacteria in the GIT. A total of $93 \%$ of patients with ESBL- and AmpC-positive Enterobacteriaceae in their GIT had been hospitalized in the preceding 6 months. Most frequently, ESBL-positive strains of Klebsiella pneumoniae and AmpC-positive strains of Citrobacter freundii were identified. The ESBL enzymes were mainly of the CTX-M type. This is consistent with data from other studies confirming changes in the prevalence of broad-spectrum beta-lactamases and prevailing strains producing CTX-M as compared with bacteria with TEM and SHV enzymes ${ }^{22,23}$. A high proportion (81\%) of strains producing CTX-M types of beta-lactamases among ESBL-positive bacteria isolated from rectal swabs taken from patients with hematological malignancies was also reported by Arnan et al. ${ }^{11}$. A group of isolates in this Spanish study comprised strains with CTX-M-9 and CTX-M-1 enzymes, as opposed to our study that detected only bacteria producing beta-lactamases from the CTX-M-1 group ${ }^{11}$. Calatayud et al. analyzed rectal swabs from cancer patients and identified ESBLpositive bacteria with genes for CTX-M-9 and CTX-M-1 beta-lactamases ${ }^{20}$.

In our study, the AmpC enzymes were most frequently of types CIT and DHA. In contrast to ESBL, intestinal colonization due to AmpC-producing Enterobacteriaceae in patients with hematological malignancies has not been studied yet. However, Korona-Glowniak et al. obtained 3 AmpC-positive isolates of Enterobacteriaceae from patients with chronic lymphocytic leukemia testing the upper respiratory tract colonization of these hematological patients ${ }^{24}$. Gudiol et al. documented that Gramnegative bloodstream infections in neutropenic cancer patients were associated with high rates of multiresistant Enterobacteriaceae, including AmpC-positive Enterobacter cloacae $^{25}$.

An important task of today's clinical microbiology is to determine potentially identical selected multiresistant bacteria in order to define their likely horizontal clonal spread. It must be noted that this study identified identical strains in two pairs of patients, clearly suggesting interpersonal transmission. However, most isolates had unique restriction profiles. Thus, no clinically significant horizontal spread of identical strains was demonstrated. A small percentage of identical strains and a large diversity of ESBL-positive strains isolated from cancer patients' rectal swabs were also reported by Calatayud et al. ${ }^{20}$.

The danger of ESBL- and AmpC-positive Enterobacteriaceae in the normal microflora rests on two aspects. First, they are a source of resistance genes for other bacteria. Second, they are potential etiological agents whose high resistance to antibiotic therapy may lead to a failure of initial antibiotic therapy and thus to higher morbidity and mortality rates. Arnan et al. found no clinical significance of ESBL-positive strains of Escherichia coli colonizing the GIT of patients with hematological 
malignancies and do not consider monitoring of these strains in the GIT as useful ${ }^{11}$. However, our study identified two patients diagnosed with urinary tract and bloodstream infections caused by ESBL- or AmpC-positive Enterobacteriaceae contained in the GIT microflora. This is evidence that these strains may act as agents causing bacterial complications.

\section{ACKNOWLEDGEMENT}

Supported by the grant projects LF_2013_012 and VZ MSMT CR 6198959205. The infrastructural part of this project (Institute of Molecular and Translational Medicine) was supported by the Operational Programme Research and Development for Innovations (project CZ.1.05/2.1.00/01.0030).

Authorship contributions: MK: principal investigator and primary responsibility for the paper; MK, MHS, VP, MR, MS: manuscript writing; RS, KI: data collection; MHS, VP, MR, MS, JN: laboratory work.

Conflict of interest statement: The authors state that there are no conflicts of interest regarding the publication of this article.

\section{REFERENCES}

1. Viscoli C, EORTC International Antimicrobial Therapy Group. Management of infection in cancer patients. Studies of the EORTC International Antimicrobial Therapy Group (IATG). Eur J Cancer 2002;38(Supp.4):S82-7.

2. Mikulska M, Del Bono V, Raiola AM, Bruno B, Gualandi F, Occhini D, di Grazia C, Frassoni F, Bacigalupo A, Viscoli C. Blood stream infections in allogeneic hematopoietic stem cell transplant recipients: reemergence of Gram-negative rods and increasing antibiotic resistance. Biol Blood Marrow Transplant 2009;15(1):47-53.

3. Trecarichi EM, Tumbarello M, Caira M, Candoni A, Cattaneo C, Pastore D, Fanci R, Nosari A, Vianelli N, Busca A, Spadea A, Pagano L. Multidrug resistant Pseudomonas aeruginosa bloodstream infection in adult patients with hematologic malignancies. Haematologica 2011;96(1):e1-e3.

4. Cattaneo C, Quaresmini G, Casari S, Capucci MA, Micheletti M, Borlenghi E, Signorini L, Re A, Carosi G, Rossi G. Recent changes in bacterial epidemiology and the emergence of fluoroquinoloneresistant Escherichia coli among patients with haematological malignancies: results of a prospective study on 823 patients at a single institution. J Antimicrob Chemother 2008;61(3):721-8.

5. Kolar M, Htoutou Sedlakova $\mathrm{M}$, Hanulik V and working group. Multiresistant Gram-negative bacteria in haemato-oncologic patients. Postgrad Med 2012;14(5):6-10.

6. Livermore DM. Fourteen years in resistance. Int J Antimicrob Agents 2012;39(4):283-94.

7. Bush K, Jacoby GA. Updated Functional Classification of $\beta$-Lactamases. Antimicrob Agents Chemother 2010;54(3):969-76.

8. Toussaint E, Bahel-Ball E, Vekemans M, Georgala A, Al-Hakak L, Paesmans M, Aoun M. Causes of fever in cancer patients (prospective study over 477 episodes). Support Care Cancer 2006;14(7):763-9.

9. Kang Cl, Chung DR, Ko KS, Peck KR, Song JH, Korean Network for Study of Infectious Diseases. Risk factors for infection and treatment outcome of extended-spectrum $\beta$-lactamase-producing Escherichia coli and Klebsiella pneumoniae bacteremia in patients with hematologic malignancy. Ann Hematol 2012;91(1):115-21.
10. Satlin MJ, Calfee DP, Chen L, Fauntleroy KA, Wilson SJ, Jenkins SG, Feldman EJ, Roboz GJ, Shore TB, Helfgott DC, Soave R, Kreiswirth BN, Walsh TJ. Emergence of carbapenem-resistant Enterobacteriaceae as causes of bloodstream infections in patients with hematologic malignancies. Leuk Lymphoma 2013;54(4):799-806.

11. Arnan M, Gudiol C, Calatayud L, Liñares J, Dominguez MÁ, Batlle M, Ribera JM, Carratalà J, Gudiol F. Risk factors for, and clinical relevance of, faecal extended-spectrum $\beta$-lactamase producing Escherichia coli (ESBL-EC) carriage in neutropenic patients with haematological malignancies. Eur J Clin Microbiol Infect Dis 2011;30(3):335-60.

12. Schaumann R, Knoop N, Genzel GH, Losensky K, Rosenkranz C, Stîngu CS, Schellenberger W, Rodloff AC, Eschrich K. A step towards the discrimination of beta-lactamase-producing clinical isolates of Enterobacteriaceae and Pseudomonas aeruginosa by MALDI-TOF mass spectrometry. Med Sci Monit 2012;18(4):MT71-7.

13. Htoutou Sedlakova M, Hanulik V, Chroma M, Hricova K, Kolar M, Latal T, Schaumann R, Rodloff AC. Phenotypic detection of broad-spectrum beta-lactamases in microbiological practice. Med Sci Monit 2011;17(5):BR147-52.

14. Chanawong A, M'Zali FH, Heritage J, Lulitanond A, Hawkey PM. Characterisation of extended-spectrum B-lactamases of the SHV family using a combination of PCR-single strand conformational polymorphism (PCR-SSCP) and PCR-restriction fragment length polymorphism (PCR-RFLP). FEMS Microbiol Lett 2000;184(1):85-9.

15. Arlet $G$, Brami $G$, Décrè $D$, Flippo $A$, Gaillot $O$, Lagrange $P H$, Philippon A. Molecular characterisation by PCR-restriction fragment length polymorphism of TEM $\beta$-lactamases. FEMS Microbiol Lett 1995;134(2-3):203-8.

16. Pagani L, Dell'Amico E, Migliavacca R, D'Andrea MM, Giacobone E, Amicosante G, Romero E, Rossolini GM. Multiple CTX-M-type extended-spectrum $\beta$-lactamases in nosocomial isolates of Enterobacteriaceae from a hospital in northern Italy. J Clin Microbiol 2003;41(9):4264-9.

17. Minarini LA, Gales AC, Palazzo IC, Darini AL. Prevalence of community-occurring extended spectrum b18. Pérez-Pérez FJ, Hanson ND. Detection of plasmid-mediated AmpC $\beta$-lactamase genes in clinical isolates by using multiplex PCR. J Clin Microbiol 2002;40(6):2153-62.

19. Husickova V, Cekanova L, Chroma M, Htoutou-Sedlakova M, Hricova K, Kolar M. Carriage of ESBL- and AmpC-positive Enterobacteriaceae in the gastrointestinal tract of community subjects and hospitalized patients in the Czech Republic. Biomed Pap Med Fac Univ Palacky Olomouc Czech Repub 2012;156(4):348-53.

20. Calatayud L, Arnan M, Liñares J, Dominguez MA, Gudiol C, Carratalà J, Batlle M, Ribera JM, Gudiol F. Prospective study of fecal colonization by extended-spectrum- $\beta$-lactamase-producing Escherichia coli in neutropenic patients with cancer. Antimicrob Agents Chemother 2008;52(11):4187-90.

21. Liss BJ, Vehreschild JJ, Cornely OA, Hallek M, Fätkenheuer G, Wisplinghoff $\mathrm{H}$, Seifert $\mathrm{H}$, Vehreschild MJ. Intestinal colonisation and blood stream infections due to vancomycin-resistant enterococci (VRE) and extended-spectrum beta-lactamase-producing Enterobacteriaceae (ESBLE) in patients with haematological and oncological malignancies. Infection 2012;40(6):613-9.

22. Canton R, Coque TM. The CTX-M beta-lactamase pandemic. Curr Opin Microbiol 2006;9(5):466-75.

23. Livermore DM, Canton R, Gniadkowski M, Nordmann $P$, Rossolin GM, Arlet G, Ayala J, Coque TM, Kern-Zdanowicz I, Luzzaro F, Poirel L, Woodford N. CTX-M: changing the face of ESBLs in Europe. J Antimicrob Chemother 2007;59(2):165-74.

24. Korona-Glowniak I, Grywalska E, Chudzik B, Bojarska-Junak A Malm A, Rolinski J. Upper respiratory tract colonization by gramnegative rods in patients with chronic lymphocytic leukemia: Analysis of risk factors. Scientific World Journal 2012:617218. doi: 10.1100/2012/617218.

25. Gudiol C, Bodro M, Simonetti A, Tubau F, González-Barca E, Cisnal M, Domingo-Domenech E, Jiménez L, Carratalà J. Changing aetiology, clinical features, antimicrobial resistance, and outcomes of bloodstream infection in neutropenic cancer patients. Clin Microbiol Infect 2013;19(5):474-79. 